\title{
So far so close: an insight into smart working and telehealth reorganization of a Language and Learning Disorders Service in Milan during COVID-19 pandemic
}

\author{
Daniela Sarti ${ }^{1}$ (D) Marinella De Salvatore ${ }^{1} \cdot$ Stefania Gazzola $^{1} \cdot$ Chiara Pantaleoni $^{1}$ • Elisa Granocchio ${ }^{1}$
}

Received: 27 April 2020 / Accepted: 20 May 2020 / Published online: 29 May 2020

(C) Fondazione Società Italiana di Neurologia 2020

COVID pandemic forced a large number of countries around the world to restructure the social life and the people working activities, according to the newly established rules of individual and group protection Figure 1.

Social distancing and stay-at-home orders act as the main barriers against virus spreading. They constitute, however, a substantial challenge in taking care and rehabilitation of children with neuro-development disorders, which are based in direct contact and affective resonance. Research already highlighted, in children living in pandemic affected areas, emotional and behavioral issues as a consequence of adult stress or discomfort and of contention measures [1].

It can be expected even more so in fragile children showing neuro-development disorders. Specifically, in language and learning disorders, for which the estimated incidence is 5$10 \%$ of the population, the guidelines in taking charge of these pathologies suggest early detection and ongoing treatments to lower risk factors and comorbidity.

In Italy, following government regulations on virus spread containment (March 9 DPCM "Io resto a casa" - "I'm staying home"), child neuropsychiatric services have been forced to suspend care activities. The management adapted to this event in very different ways: forced holidays, part-time work, smart working, wage compensation (fund); in Lombardy, one of the most struck regions, a decree promoted remote work and guaranteed telepractice reimbursement by the regional health system. The Italian Society of Infantile Neuropsychiatry (SINPIA) drafted a practical document about the service reorganization (www.sinpia.eu).

Daniela Sarti

daniela.sarti@istituto-besta.it

1 Developmental Neurology Unit - Language and Learning Disorders Service, Fondazione IRCCS Istituto Neurologico Carlo Besta, Milan, Italy
The Management of Fondazione IRCCS Istituto Neurologico Besta in Milan, one of the most struck town, agreed upon the request to secure continuous assistance to patients and to assure workers' protection by abiding by the rules in force, started a project of telemedicine for outpatient activities, in accordance with experiences made in Italy by other neurologists as specified in the article by Caso and Federico [2], and allowed smart working procedures for professionals involved in children diagnosis and rehabilitation, with the only exception of doctors and nurses.

Such services have been promptly reorganized, with the challenging quick design of a new way to provide services, and a number of coordination meetings were held.

\section{The reorganization of the Language and Learning Disorder Service}

Our service reorganization required an answer to the following questions:

- Which are the goals and activities of the personnel, speech therapists, psychologists, and child neuropsychiatrists?: rehabilitation, counseling, parents' interviews, assessments.

- Which is the best way to provide services and information and communication technology (ICT) tools?: telerehabilitation with commonly available platforms; experimental trainings; word forms; clinical interviews in conference call; direct child video-assessment or via video clips or by filling forms. Other tools are as follows: email, phone, video calls.

- How to record activities?: recording of all activities and performing services in a shared centralized calendar, remotely accessible from home

- How and when the activities have to be reviewed?: monthly verifications against defined goals and objectives 


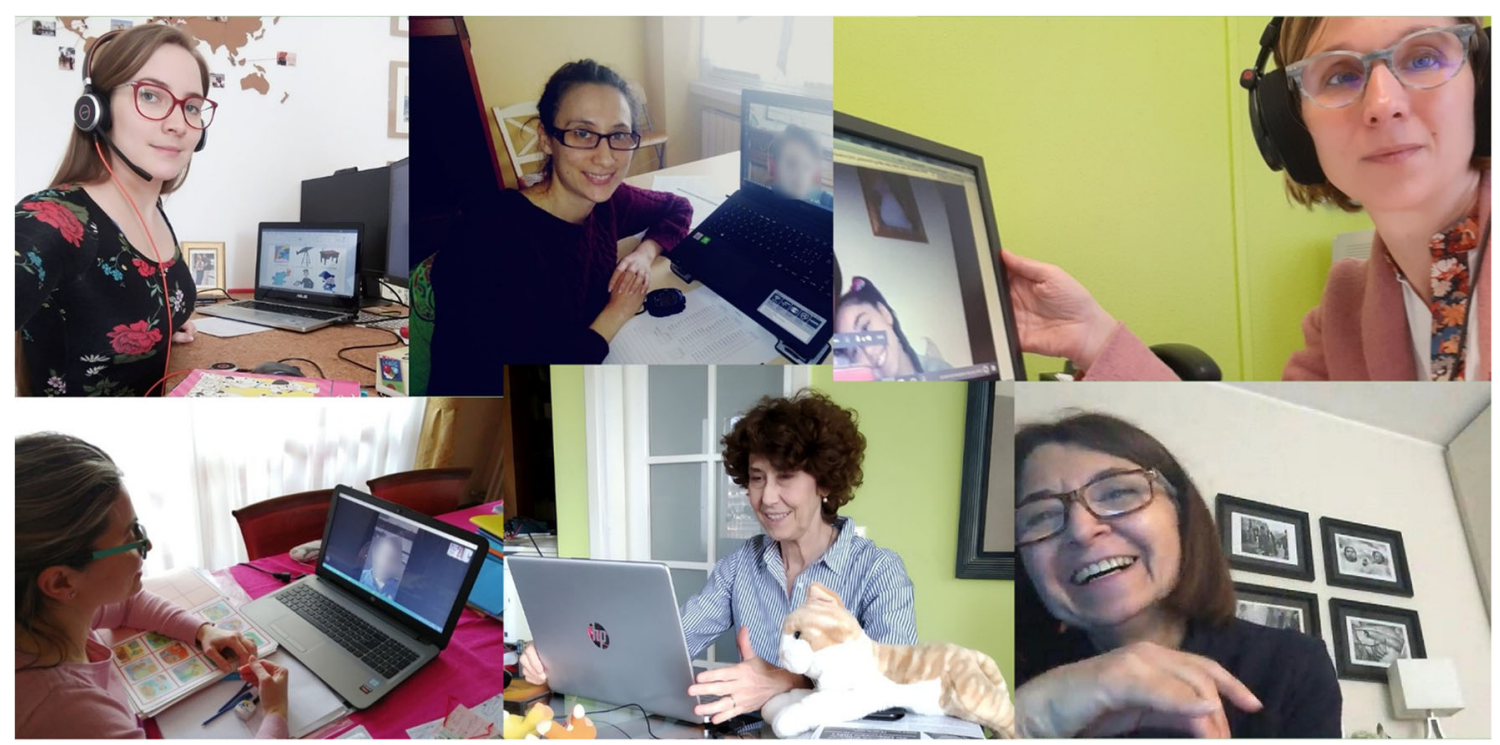

Fig. 1. Our task force in home setup

- Running verifications?: yes, the smart working coordinator keeps tabs with all operators through the platform and coordinates the workers in the hospital to check the feasibility and effectiveness of the tele-rehab and assessment activities

- How to share patient data?: smart workers and in-hospital workers share a common database of patients' directory and contacts.

To redefine the therapeutic protocol in terms of tele-working, the professionals check the feasibility of a tele-assessment and tele-rehabilitation of children according to an informative check-list:

- Child features (age, diagnosis, severity level, physical and sensorial features, cognitive-behavioral profile, communicative and language skills)

- Family compliance (PC and software availability, suitable spaces at home, parents' cooperation level, outstanding elearning requests)

A new plan to take charge and to rehabilitate is hence drafted with the parents.

\section{Strengths and weaknesses}

Timeliness of reorganization and the effective adaptation to the challenge shown by the personnel and family; research and creativity in reinventing methods and materials, respecting principles and ethics. This way, it became possible to follow-up on the treatment of all children already in charge by the service.
- for scholar children with learning disabilities, by using online dyslexia platforms and editing audio-visual materials for dysorthography and dyscalculia;

- for preschooler children with language disorders, by using online language impairment platforms and training with audio-visual materials;

- for toddlers, by preparing rehabilitation monitoring, video-interviewing parents, filling up survey forms, and looking at caregivers' provided clips.

We additionally performed thorough monitoring of language and learning development by means of interviews about communicative and linguistic functioning, surveys, and observations of familiar made videos.

The smart working and telehealth reorganization has offered the potential to deliver during April 2020; after the first month of lockdown, a number of services carried out by speech therapists and psychologists equal to $85 \%$ of those realized face to face in April 2019 (291 versus 342). Those include intensive bi-triweekly treatment sessions, parent training, psychological support, and monitoring by means of structured interviews and surveys. It has offered access to speech and learning therapy services to all children already in treatment and to get to other families to offer monitoring, with a total of 40 patients.

Family response has been immediately positive and active; informal feedback from the family indicates high levels of satisfaction. The majority of parent comments reflect a positive perception of the reorganization; they reported improvements in their children's communication and learning skills as a result of tele-speech and learning therapy.

Availability of computers has not been a barrier for the families, having always a PC or a tablet; a number of families were forced to arrange schedules to share the use of the tools 
between rehabilitation and smart working and e-learning, by impacting the time tables.

Some parents appreciated the help of the therapists to solve the technological difficulties experienced during the project. However, the quality of the videoconferencing equipment and infrastructure may affect the quality and perception of telepractice services.

During the tough lockdown period, many parents expressed satisfaction with counseling held by telepractice. It provided support to emotionally manage the children and to handle e-learning requests from school.

However, the families report surfacing problems with children in this difficult moment and increasing challenges in parenting and education, even if we found also fair improvements.

Personal and social autonomy development seems to slow down and some emerging skills relax. Parents report lack of orientation due to rhythm and space changing, hyperactivity, mood fluctuations, anger and aggressiveness, and reduced ability to withstand failures [1]. The perceived level and frequency varies according to neurodevelopmental disorder.

As more and more resources are being directed toward telepractice, it is critical to assess the satisfaction level among service providers, caregivers, and children. Specific information about what was desirable and what was unsatisfactory about telepractice would be helpful to learn, so we are developing a telehealth satisfaction survey to be carried out by all patients and parents.

Nowadays, there is a spreading trend of promoting the application of telecommunication technology in many fields, including neurodevelopmental disorder services for assessment, intervention, and/or consultation. In Italy, so far, clinicians made a limited use of technology during their clinical practice. There are also few researches about validated assessments for teleadministration of tests or comparing the validity of in-person versus remote assessment protocols for childhood language and learning disorders, necessary for telehealth to become an accepted alternative mode of service provisioning [3, 4]. However, during the epidemic, this evolution has been boldly accelerated in Italy as testified by other reports recently published about several neurological conditions in this journal $[5,6]$.

This represents one of the challenges for researchers, policymakers, and speech-language pathologists to more successfully use new technologies for assessment and intervention and to develop more targeted initiatives that better support young people, caregivers, and service providers by use of the Internet and different information and communication technologies.

\section{Conclusion}

Telehealth has the potential to improve children's access to speech-language and learning pathology services especially during COVID-19 emergency and also within the time period that will follow the pandemic, when social relations will get back to normal, but very slowly and possibly not really as before.

In preparation for a gradual reduction of lockdown measures, we need to honestly evaluate the opportunity to restart face-to-face services. Alternatively, we can continue to provide remote services and move forth the reestablishment of normal activities.

This choice could be supported by the clinical and epidemiological about COVID-19 in children. These data indicate that children are less frequently affected (1.5\% of cases) and generally have milder symptoms but they show a persistent positive viral excretion from the gastrointestinal tract after they had recovered [7]. Furthermore, there is an increased risk of critical disease and mortality in children with underlying comorbidities, including chronic neurological disorders.

Hence, the risk of infection in children attending neuropsychiatric services could become very high for patients and operators; it should be needed special management of children with pre-existing chronic conditions [8] and all choices should be driven by the need to secure continuous assistance to patients and to assure workers' protection.

Smart working may foster a valuable occasion, for all professionals, to develop new strategies and means to deliver assistance and services. The expanding role of telecommunication technologies may support the process of the guidelines development for practice in this area, intended to ease the continued systematic improvement and to help ensuring a high level of professional practice by psychologists, physicians, and speech pathologists especially in this emergency.

According to Caso and Federico [2], the epidemic gives the occasion to develop and improve the care organization; in particular, our experience could be useful to evaluate the feasibility of an emergency response model for the assistance and rehabilitation of children with neurodevelopmental disabilities as language and learning pathology.

Acknowledgments The authors are grateful to Dr. Claudia Ciaccio for support in English editing.

\section{Compliance with ethical standards}

Conflict of interest None.

Ethical approval None.

\section{References}

1. Jiao WY, Wang LN, Liu J, Fang SF, Jiao FY, Pettoello-Mantovani M, Somekh E (2020) Behavioral and emotional disorders in children during the COVID-19 epidemic. J Pediatr 221:264-266.e1. https:// doi.org/10.1016/j.jpeds.2020.03.013 [Epub ahead of print] 
2. Caso V, Federico A (2020) No lockdown for neurological diseases during COVID19 pandemic infection. Neurol Sci 41(2020):9991001. https://doi.org/10.1007/s10072-020-04389-3

3. Sutherland R, Trembath D, Hodge A, Drevensek S, Lee S, Silove N, Roberts J (2017) Telehealth language assessments using consumer grade equipment in rural and urban settings: feasible, reliable and well tolerated. J Telemed Telecare 23(1):106-115. https://doi.org/10. $1177 / 1357633 X 15623921$

4. Grogan-Johnson S, Schmidt AM, Schenker J, Alvares R, Rowan LE, Taylor J (2013) A comparison of speech sound intervention delivered by telepractice and side-by-side service delivery models. Commun Disord Q 34(4):210-220. https://doi.org/10.1177/ 1525740113484965

5. Baracchini C, Pieroni A, Viaro F, Cianci V, Cattelan AM, Tiberio I et al (2020) Acute stroke management pathway during Coronavirus19 pandemic. Neurol Sci 41(2020):1003-1005. https://doi.org/10. 1007/s10072-020-04375-9
6. Bonavita S, Tedeschi G, Atreja A, Lavorgna L (2020) Digital triage for people with multiple sclerosis in the age of COVID-19 pandemic. Neurol Sci 41(2020):1007-1009. https://doi.org/10.1007/s10072020-04391-9

7. Xu Y, Li X, Zhu B, Liang H, Fang C, Gong Y et al (2020) Characteristics of pediatric SARS-CoV-2 infection and potential evidence for persistent fecal viral shedding. Nat Med 26:502-505. https://doi.org/10.1038/s41591-020-0817-4

8. Dayal D (2020) We urgently need guidelines for managing COVID19 in children with comorbidities. Acta Paediatr. https://doi.org/10. 1111/apa.15304 [Epub ahead of print]

Publisher's note Springer Nature remains neutral with regard to jurisdictional claims in published maps and institutional affiliations. 